J. Clin. Chem. Clin. Biochem.

Vol. 23, 1985, pp. 287-292

\title{
Interference by Analgesic and Antirheumatic Drugs in 25 Common Laboratory Assays
}

\author{
By Z. Jelić-Ivanović, N. Majkić-Singh, S. Spasić, P. Todorović and D. Živanov-Stakić \\ Department of Biochemistry, Faculty of Pharmacy, Belgrade, Yugoslavia
}

(Received September 26/December 10, 1984)

Summary: Twenty five different analytical procedures, commonly used in clinical laboratories, were investigated for interference by eight analgesic and antirheumatic drugs. Ten of the investigated assays showed no statistically significant interference.

Acetylsalicylic acid interfered in six assays (for glucose, uric acid, protein and cholesterol). Aminophenazone significantly decreased glucose, bilirubin and protein values, whereas caffeine affected four methods (for glucose, protein and iron).

No definite influence of phenobarbital could be detected on any assay. Glucose, uric acid and iron values were altered in the presence of diclofenac. Indomethacin interfered in glucose, urea, uric acid and protein assays.

Samples containing ibuprofen had altered creatinine, bilirubin and iron values, whereas ketoprofen interfered in glucose and iron determination.

Untersuchung von 25 üblichen Laboratoriumsmethoden hinsichtlich ihrer Störanfälligkeit durch Analgetica und Antirheumatica.

Zusammenfassung: Fünfundzwanzig verschiedene analytische, in klinischen Laboratorien übliche Verfahren wurden hinsichtlich ihrer Störanfälligkeit durch acht Analgetica und Antirheumatica untersucht. Zehn dieser Methoden zeigten keine statistisch signifikante Störung.

Acetylsalicylsäure störte bei sechs Verfahren zur Bestimmung von Glucose, Harnsäure, Protein und Cholesterin. Aminophenazon verminderte Glucose-, Bilirubin- und Proteinwerte signifikant, während Coffein vier Methoden zur Bestimmung von Glucose, Protein und Eisen störte.

Phenobarbital war ohne jeglichen Einfluß auf die untersuchten Methoden. Glucose-, Harnsäure- und Eisenwerte waren in Gegenwart von Diclofenac verändert. Indomethacin störte Glucose-, Harnstoff-, Harnsäureund Proteinbestimmungen. Ibuprofen enthaltende Proben ergaben veränderte Kreatinin-, Bilirubin- und Eisenwerte, während Ketoprofen bei Glucose- und Eisenbestimmungen störte.

\section{Introduction}

In our previous investigation, effects of analgesic and antirheumatic drugs on the assay of serum enzymes (1) and SMA II procedures (2) were examined.

Acetylsalicylic acid, aminophenazone, indomethacin, diclofenac, ibuprofen and ketoprofen are widely used

in the treatment of various rheumatic diseases. Analgetic preparations containing acetylsalicylic acid, aminophenazone, phenobarbital or caffeine are also frequently used in self-medication. It is thus important to know whether these drugs alter clinical laboratory test results. 
In the present work, we investigated the effects of these eight drugs (in vitro) on 25 of the most frequently used methods for the determination of glucose, urea, uric acid, creatinine, bilirubin, protein, cholesterol, triglycerides and iron.

Tab. 1. Concentrations of added drugs in reconstituted lyophilized human sera.

\begin{tabular}{ll}
\hline Drug & $\begin{array}{l}\text { Concen- } \\
\text { tration } \\
(\mu \mathrm{mol} / \mathrm{l})\end{array}$ \\
\hline $\begin{array}{l}\text { Acetylsalicylic acid } \\
\quad \text { 2-(acetyloxy)benzoic acid] }\end{array}$ & 8326 \\
$\begin{array}{l}\text { Aminophenazone (4-dimethylamino- } \\
\text { 2,3-dimethyl-1-phenyl-3 pyrazolin-5-one) }\end{array}$ & 540 \\
$\begin{array}{l}\text { Caffeine (1,3,7-trimethylxanthine) } \\
\text { Phenoarbital [5-ethyl,5-phenyl-2,4,6(1H, } \\
\text { 3H, 5H)-pyrimidinetrione] }\end{array}$ & 772 \\
$\begin{array}{l}\text { Ibuprofen [ } \alpha \text {-methyl-4-(2-methylpropyl)- } \\
\text { benzenacetic acid] }\end{array}$ & 1076 \\
$\begin{array}{l}\text { Diclofenac, 2-[(2,6-dichlorophenyl)-amino]- } \\
\text { benzenacetic acid }\end{array}$ & 970 \\
$\begin{array}{l}\text { Indomethacin [1-(p-chlorobenzoyl)- } \\
\text { 5-methoxy-2-methylindole-3-acetic acid] }\end{array}$ & 71 \\
$\begin{array}{l}\text { Ketoprofen [2-(3-benzoylphenyl)- } \\
\text { propionic aicd] }\end{array}$ & 35 \\
\hline
\end{tabular}

\section{Materials and Methods}

To examine the drug interferences in common laboratory assays, the general protocol by Siest et al. (3) was followed. Commercial lyophilized human sera were reconstituted with solutions of each drug tested. The drug concentrations (tab. 1) were approximately ten times the maximal therapeutic levels $(4-7)$.

Aqueous solutions of acetylsalicylic 'acid, aminophenazone, caffeine, phenobarbital, diclofenac and indomethacin were made. For control sample preparation, the drug solutions were replaced by distilled water. To dissolve ibuprofen and ketoprofen, ethanol was added in a final concentration of $200 \mathrm{~g} / \mathrm{l}$, and additional control samples were prepared, containing the same concentration of ethanol.

Glucose, urea, uric acid, creatinine, bilirubin, protein, cholesterol, triglyceride and iron concentrations were determined by various methods commonly used in clinical laboratories. The principles and reference data of the methods are shown in table 2.

For each method, six replicate tests were performed on control samples and samples containing the drug; the statistical parameters: mean value $(\overline{\bar{x}})$ and standard deviation (s) were determined. Using Student's t-test, the significance of the differences between values obtained in sera with and without drugs was assessed. All tests for a specific group of samples (sera con= taining the drug and corresponding controls) were completed in a single run.

The interferences observed were studied further using lower drug concentrations down to the therapeutic levels. Concentrations of drugs in samples were gradually decreased and drug effects tested, until statistically unsignificant differences were found.

The assays were performed with a Pye Unicam spectrophotometer SP 8-100.

Tab. 2. The methods used for the determination of analytes.

\begin{tabular}{|c|c|c|c|}
\hline Analyte & Method & & Reference \\
\hline Glucose & $\begin{array}{l}\text { Hexokinase and glucose-6-phosphate dehydrogenase } \\
\text { Glucose dehydrogenase } \\
\text { Glucose oxidase and peroxidase, using 4-aminophenazone and } \\
2,4 \text {-dichlorphenol as chromogen } \\
\text { Glucose oxidase and peroxidase, using ABTS as chromogen } \\
\text { o-Toluidine }\end{array}$ & & $\begin{array}{l}8 \\
9 \\
10 \\
11 \\
12\end{array}$ \\
\hline Urea & $\begin{array}{l}\text { Urease and glutamate dehydrogenase } \\
\text { Phenol-hypochlorite } \\
\text { Diacetylmonoxime }\end{array}$ & & $\begin{array}{l}1 \overline{3} \\
14 \\
15\end{array}$ \\
\hline Uric acid & $\begin{array}{l}\text { Uricase and catalase } \\
\text { Uricase, catalase and aldehyde dehydrogenase } \\
\text { Direct UV-test with uricase }\end{array}$ & & $\begin{array}{l}16 \\
17 \\
18\end{array}$ \\
\hline Creatinine & $\begin{array}{l}\text { Continuous, with alkaline picrate } \\
\text { Alkaline picrate, with deproteinization } \\
\text { Slot }\end{array}$ & & $\begin{array}{l}20 \\
21 \\
22\end{array}$ \\
\hline Bilirubin & $\begin{array}{l}\text { Jendrassik-Gróf } \\
\text { Dimethylsulphoxide } \\
\text { Direct spectrophotometric method (at } 461 \text { and } 551 \mathrm{~nm} \text { ) }\end{array}$ & & $\begin{array}{l}23 \\
24 \\
25\end{array}$ \\
\hline Protein & $\begin{array}{l}\text { Biuret reaction } \\
\text { Direct spectrophotometric method (at } 280 \mathrm{~nm} \text { ) }\end{array}$ & & $\begin{array}{l}26 \\
27\end{array}$ \\
\hline Cholesterol & $\begin{array}{l}\text { Enzymatic, with cholesterol esterase and cholesterol oxidase } \\
\text { Liebermann-Burchard reaction }\end{array}$ & & $\begin{array}{l}28 \\
29\end{array}$ \\
\hline Triglycerides & $\begin{array}{l}\text { Enzymatic, using lipase, glycerol kinase, pyruvate kinase and } \\
\text { lactate dehydrogenase }\end{array}$ & & 30 \\
\hline Iron & $\begin{array}{l}\text { Bathophenanthroline disulphonate } \\
\alpha, \alpha^{\prime} \text {-Dipyridyl (Ramsay) }\end{array}$ & - & $\begin{array}{l}31 \\
32\end{array}$ \\
\hline
\end{tabular}




\section{Results and Discussion}

The effects of analgesic and antirheumatic drugs on 25 common laboratory assays are shown in tables 3-5. The concentrations of analytes and their variations obtained using different methods in samples with and without the drugs are given, as well as the corresponding $p$ values. Ten assays showed no statistically significant interference, i.e. the determination of glucose (hexokinase method), urea (with glutamate dehydrogenase and phenol-hypochlorite method), uric acid (direct UV test and phosphotungstate method), creatinine (continuous and Slot's method), bilirubin (Jendrassik-Gróf), cholesterol and triglycerides (enzymatic assays). No definite influence of phenobarbital could be detected on any method (tab. 4).

Acetylsalicylic acid interfered in six assays (for glucose, uric acid, protein and cholesterol determination) (tab. 3). Out of five glucose assays tested, two were affected by the drug: the glucose dehydrogenase method and the glucose oxidaseperoxidase procedure using ABTS as chromogen. Acetylsalicylic acid has been reported to have no in vitro effect on the alkaline ferricyanide, $p$-HBAH and $o$-toluidine methods (33). In sera containing the drug, apparent concentrations of uric acid (with uricase and catalase) and protein (direct spectrophotometric assay) were very significantly increased ( $p<0.01$ and $\mathrm{p}<0.005$, respectively). Wirth \& Thompson (34) reported interference by acetylsalicylic acid in the FolinCiocalteu protein assay in body fluids. Lower cholesterol values were obtained using the LiebermannBurchard method ( $\mathrm{p}<0.01)$, which is in agreement with findings reported by Caraway \& Kammeyer (35).

Aminophenazone significantly decreased the results for glucose (with ABTS, $\mathrm{p}<0.001$ ), bilirubin (with dimethylsulphoxide, $\mathrm{p}<0.05$ ), and protein (biuret method, $p<0.05$ ). The drug is also known to increase the results of the cholesterol determination based on reaction with ferric ions in acetic acid-sulphuric acid $(33,35)$.

Tab. 3.Effects of acetylsalicylic acid, aminophenazone and caffeine on some common laboratory tests $(\mathrm{N}=6$, number of the degrees of freedom $\emptyset=2 \mathrm{~N}-2=10$ ).

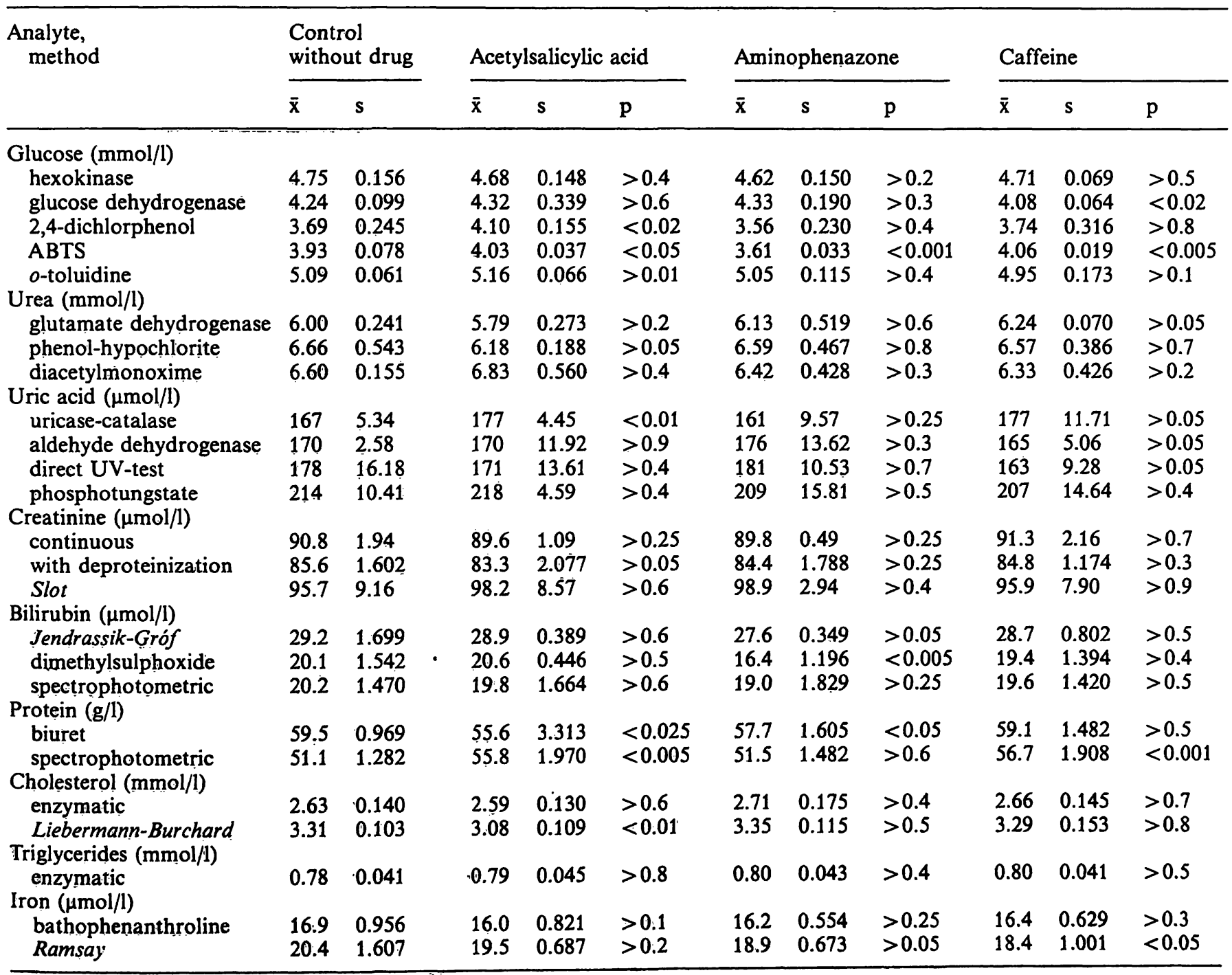


Caffeine affected four tests (tab. 3). Glucose values were very significantly increased (ABTS method, $p<0.005$ ), as well as protein (spectrophotometric assay, $p<0.001$ ). Interference by caffeine in the uric acid assay using phosphotungstate has been reported (35), but the effect was found to be significant only in the determination of uric acid in urine.

Effects of diclofenac and indomethacin are shown in table 4. In samples containing these drugs, the values for glucose (with ABTS) and uric acid values (with uricase and catalase and with aldehyde dehydrogenase) were elevated. Indomethacin affected very significantly the results of the glucose determination (with glucose dehydrogenase, $p<0.005$ ), and the protein determination (biuret method, $\mathrm{p}<0.005$ ).

Creatinine (with deproteinization) and iron (Ramsay) values were increased in sera containing ibuprofen (tab. 5). Ketoprofen interfered in glucose (with ABTS, $p<0.02$ ) and iron assays (with bathophenanthroline, $\mathrm{p}<0.05$ and Ramsay method, $\mathrm{p}<0.005$ ). No data have been previously reported on analytical interferences by diclofenac, indomethacin and ketoprofen.

The effects found were studied further using lower concentrations of drugs, down to the therapeutic levels. Interference by aminophenazone in the glucose determination (with ABTS) was still evident at $216 \mu \mathrm{mol}$ of drug per liter $(\mathrm{p}<0.025)$. Lower concentration of diclofenac $(28 \mu \mathrm{mol} / \mathrm{l})$ significantly increased the results of the same test $(p<0.05)$. Figure 1 shows the dose dependence of these two interferences. In lower concentrations, other drugs did not interfere with any assay, so the effects found are significant only in cases of drug overdosage or poisoning.

Tab. 4. Phenobarbital, diclofenac and indomethacin interference in common laboratory assays $(\mathrm{N}=6$, number of the degrees of freedom $\emptyset=10$ ).

\begin{tabular}{|c|c|c|c|c|c|c|c|c|c|c|c|}
\hline \multirow[t]{2}{*}{$\begin{array}{l}\text { Analyte, } \\
\text { method }\end{array}$} & \multicolumn{2}{|c|}{$\begin{array}{l}\text { Control } \\
\text { without drug }\end{array}$} & \multicolumn{3}{|c|}{ Phenobarbital } & \multicolumn{3}{|c|}{ Diclofenac } & \multicolumn{3}{|c|}{ Indomethacin } \\
\hline & $\overline{\mathbf{x}}$ & $\mathbf{s}$ & $\overline{\mathbf{x}}$ & $\mathbf{s}$ & $\mathrm{p}$ & $\overline{\mathbf{x}}$ & $\mathbf{s}$ & $\mathrm{p}$ & $\overline{\mathbf{x}}$ & $\mathbf{s}$ & $\mathrm{p}$ \\
\hline \multicolumn{12}{|l|}{ Glucose (mmol/l) } \\
\hline hexokinase & 4.75 & 0.156 & 4.88 & 0.215 & $>0.3$ & 4.84 & 0.276 & $>0.5$ & 4.76 & 0.108 & $>0.9$ \\
\hline glucose dehydrogenase & 4.24 & 0.099 & 4.26 & 0.067 & $>0.6$ & 4.16 & 0.139 & $>0.3$ & 4.44 & 0.071 & $<0.005$ \\
\hline 2,4-dichlorphenol & 3.69 & 0.245 & 3.84 & 0.167 & $>0.3$ & 3.87 & 0.079 & $>0.1$ & 3.71 & 0.232 & $>0.3$ \\
\hline ABTS & 3.93 & 0.078 & 4.00 & 0.084 & $>0.1$ & 4.11 & 0.059 & $<0.005$ & 4.05 & 0.073 & $<0.05$ \\
\hline$o$-toluidine & 5.09 & 0.061 & 5.00 & 0.086 & $>0.05$ & 5.15 & 0.057 & $>0.1^{\cdots}$ & 5.18 & 0.047 & $<0.05$ \\
\hline \multicolumn{12}{|l|}{ Urea (mmol/l) } \\
\hline glutamate dehydrogenase & 6.00 & 0.241 & 6.10 & 0.192 & $>0.4$ & 6.24 & 0.128 & $>0.05$ & 6.23 & 0.352 & $>0.2$ \\
\hline phenol-hypochlorite & 6.66 & 0.543 & 6.60 & 0.310 & $>0.08$ & 6.32 & 0.450 & $>0.3$ & 6.49 & 0.328 & $>0.5$ \\
\hline diacetylmonoxime & 6.60 & 0.155 & 6.39 & 0.199 & $>0.3$ & 6.77 & 0.563 & $>0.5$ & 6.29 & 0.169 & $>0.02$ \\
\hline \multicolumn{12}{|l|}{ Uric acid $(\mu \mathrm{mol} / \mathrm{l})$} \\
\hline uricase-catalase & 167 & 5.34 & 171 & 8.31 & $>0.3$ & 180 & 8.19 & $<0.02$ & 179 & 9.18 & $<0.05$ \\
\hline aldehyde dehydrogenase & 170 & 2.58 & 165 & 6.37 & $>0.1$ & 195 & 9.02 & $<0.001$ & 183 & 9.70 & $<0.02$ \\
\hline direct UV-test & 178 & 16.18 & 182 & 9.51 & $>0.6$ & 176 & 3.30 & $>0.7$ & 184 & 17.20 & $>0.5$ \\
\hline phosphotungstate & 214 & 10.41 & 217 & 12.10 & $>0.6$ & 212 & 7.81 & $>0.8$ & 222 & 17.87 & $>0.3$ \\
\hline \multicolumn{12}{|l|}{ Creatinine $(\mu \mathrm{mol} / \mathrm{l})$} \\
\hline continuous & 90.8 & 1.94 & 90.7 & 0.75 & $>0.8$ & 89.5 & 2.38 & $>0.3$ & 88.7 & 1.47 & $>0.05$ \\
\hline with deproteinization & 85.6 & 1.602 & 85.4 & 0.767 & $>0.8$ & 84.1 & 1.376 & $>0.1$ & 84.7 & 1.591 & $>0.3$ \\
\hline Slot & 95.7 & 9.16 & 95.4 & 8.96 & $>0.9$ & 100.1 & 9.87 & $>0.4$ & 101.2 & 9.08 & $>0.3$ \\
\hline \multicolumn{12}{|l|}{ Bilirubin $(\mu \mathrm{mol} / \mathrm{l})$} \\
\hline Jendrassik-Gróf & 29.2 & 1.699 & 29.9 & 0.609 & $>0.8$ & 28.4 & 0.841 & $>0.3$ & 29.4 & 0.982 & $>0.8$ \\
\hline dimethylsulphoxide & 20.1 & 1.542 & 20.7 & 1.232 & $>0.5$ & 18.6 & 1.774 & $>0.1$ & 18.8 & 1.554 & $>0.2$ \\
\hline $\begin{array}{l}\text { spectrophotometric } \\
\text { Protein }(\mathrm{g} / \mathrm{l})\end{array}$ & 20.2 & 1.470 & 19.0 & 1.471 & $>0.2$ & 20.8 & 0.914 & $>0.4$ & 21.5 & 1.255 & $>0.1$ \\
\hline biuret & 59.5 & 0.969 & 57.9 & 2.607 & $>0.1$ & 60.9 & 2.738 & $>0.25$ & 57.8 & 0.568 & $<0.005$ \\
\hline spectrophotometric & 51.1 & 1.282 & 49.9 & 2.282 & $>0.3$ & 51.4 & 2.083 & $>0.8$ & 52.0 & 3.144 & $>0.05$ \\
\hline \multicolumn{12}{|l|}{ Cholesterol (mmol/l) } \\
\hline $\begin{array}{l}\text { enzymatic } \\
\text { Liebermann-Burchard }\end{array}$ & 2.63 & 0.140 & 2.58 & 0.164 & $>0.6$ & 2.62 & 0.125 & $>0.8$ & 2.63 & 0.211 & $>0.9$ \\
\hline $\begin{array}{l}\text { Liebermann-Burchard } \\
\text { Triglycerides (mmol/l) }\end{array}$ & 3.31 & 0.103 & 3.18 & 0.102 & $>0.25$ & 3.32 & 0.175 & $>0.9$ & $3: 25$ & 0.151 & $\cdot>0.4$ \\
\hline $\begin{array}{c}\text { enzymatic } \\
\text { Iron }(\mu \mathrm{mol} / \mathrm{l})\end{array}$ & 0.78 & 0.041 & 0.80 & 0.055 & $>0: 5$ & 0.79 & 0.039 & $>0.8$ & 0.78 & 0.035 & $>0.9$ \\
\hline bathophenanthroline & 16.9 & 0.956 & 16.4 & 1.029 & $>0.5$ & 16.4 & 0.835 & $>0.4$ & 16.5 & 0.964 & $>0.5$ \\
\hline Ramsay & 20.4 & 1.607 & 19.2 & 0.964 & $>0.1$ & 18.4 & 0.377 & $<0.02$ & 21.0 & 1.009 & $>0.4$ \\
\hline
\end{tabular}


Tab. 5. Effects of ibuprofen and ketoprofen on some common laboratory tests $(N=6$, number of the degrees of freedom $\emptyset=10)$.

\begin{tabular}{|c|c|c|c|c|c|c|c|c|}
\hline \multirow[t]{2}{*}{$\begin{array}{l}\text { Analyte, } \\
\text { method }\end{array}$} & \multicolumn{2}{|c|}{$\begin{array}{l}\text { Control } \\
\text { without drug }\end{array}$} & \multicolumn{3}{|c|}{ Ibuprofen } & \multicolumn{3}{|c|}{ Ketoprofen } \\
\hline & $\overline{\mathbf{x}}$ & $\mathbf{s}$ & $\overline{\mathbf{x}}$ & $\mathbf{s}$ & p & $\overline{\mathbf{x}}$ & $\mathbf{s}$ & p \\
\hline \multicolumn{9}{|l|}{ Glucose (mmol/l) } \\
\hline hexokinase & 4.83 & 0.229 & 4.95 & 0.233 & $>0.4$ & 5.02 & 0.081 & $>0.1$ \\
\hline glucose dehydrogenase & 4.36 & 0.112 & 4.21 & 0.150 & $>0.1$ & 4.30 & 0.163 & $>0.5$ \\
\hline 2,4-dichlorphenol & 4.00 & 0.061 & 4.03 & 0.108 & $>0.6$ & 4.10 & 0.184 & $>0.25$ \\
\hline ABTS & 4.10 & 0.081 & 4.11 & 0.044 & $>0.8$ & 4.26 & 0.082 & $<0.02$ \\
\hline$o$-toluidine & & 0.090 & 5.01 & 0.136 & $>0.7$ & 5.06 & 0.078 & $>0.7$ \\
\hline \multicolumn{9}{|l|}{ Urea $(\mathrm{mmol} / \mathrm{l})$} \\
\hline glutamate dehydrogenase & 6.13 & 0.278 & 6.05 & 0.324 & $>0.6$ & 6.16 & 0.107 & $>0.7$ \\
\hline phenol-hypochlorite & 6.65 & 0.353 & 6.55 & 0.582 & $>0.7$ & 6.79 & 0.556 & $>0.6$ \\
\hline diacetylmonoxime & 6.19 & 0.225 & 6.23 & 0.264 & $>0.7$ & 6.16 & 0.118 & $>0.7$ \\
\hline \multicolumn{9}{|l|}{ Uric acid $(\mu \mathrm{mol} / \mathrm{l})$} \\
\hline uricase-catalase & 178 & 8.75 & 181 & 8.60 & $>0.6$ & 174 & 11.96 & $>0.5$ \\
\hline aldehyde dehydrogenase & 172 & 9.50 & 183 & 6.02 & $>0.05$ & 178 & 14.66 & $>0.3$ \\
\hline direct UV-test & 155 & 6.53 & 160 & 9.83 & $>0.3$ & 159 & 10.29 & $>0.7$ \\
\hline phosphotungstate & 331 & 11.28 & 333 & 13.96 & $>0.7$ & 346 & 12.92 & $>0.05$ \\
\hline \multicolumn{9}{|l|}{ Creatinine $(\mu \mathrm{mol} / \mathrm{l})$} \\
\hline continuous & 92.3 & 1.94 & 92.8 & 2.31 & $>0.7$ & 92.9 & 0.57 & $>0.5$ \\
\hline with deproteinization & 88.3 & 1.357 & 91.0 & 1.010 & $>0.005$ & 86.9 & 1.668 & $>0.1$ \\
\hline Slot & 104 & 9.42 & 103 & 8.78 & $>0.9$ & 105 & 7.88 & $>0.8$ \\
\hline \multicolumn{9}{|l|}{ Bilirubin $(\mu \mathrm{mol} / \mathrm{l})$} \\
\hline Jendrassik-Gróf & 30.5 & 0.368 & 30.3 & 0.777 & $>0.5$ & 30.7 & 0.667 & $>0.6$ \\
\hline dimethylsulphoxide & 18.5 & 2.066 & 19.5 & 1.643 & $>0.3$ & 20.7 & 1.994 & $<0.05$ \\
\hline spectrophotometric & 19.4 & 0.768 & 21.3 & 1.274 & $<0.025$ & 19.2 & 1.615 & $>0.8$ \\
\hline \multicolumn{9}{|l|}{ Protein $(g / 1)$} \\
\hline biuret & 58.2 & 2.469 & 59.2 & 2.256 & $>0.25$ & 60.8 & 1.457 & $>0.05$ \\
\hline spectrophotometric & 50.2 & 2.313 & 50.6 & 1.562 & $>0.7$ & 52.3 & 2.023 & $>0.1$ \\
\hline \multicolumn{9}{|l|}{ Cholesterol (mmol/l) } \\
\hline enzymatic & 2.88 & 0.080 & 2.90 & 0.103 & $>0.6$ & 2.92 & 0.124 & $>0.4$ \\
\hline $\begin{array}{l}\text { Liebermann-Burchard } \\
\text { Triglvcerides (mmol/l) }\end{array}$ & 3.33 & 0.125 & 3.29 & 0.150 & $>0.8$ & 3.35 & 0.114 & $>0.8$ \\
\hline $\begin{array}{l}\text { Triglycerides (mmol/l) } \\
\text { enzymatic }\end{array}$ & 0.86 & 0.017 & 0.87 & 0.009 & $>0.5$ & 0.87 & 0.028 & $>0.4$ \\
\hline \multicolumn{9}{|l|}{ Iron $(\mu \mathrm{mol} / \mathrm{l})$} \\
\hline bathophenanthroline & 22.3 & 1.332 & 20.0 & 1.170 & $<0.02$ & 20.3 & 1.210 & $<0.05$ \\
\hline Ramsay & 16.6 & 0.838 & 19.6 & 0.361 & $<0.001$ & 19.49 & 1.372 & $<0.005$ \\
\hline
\end{tabular}
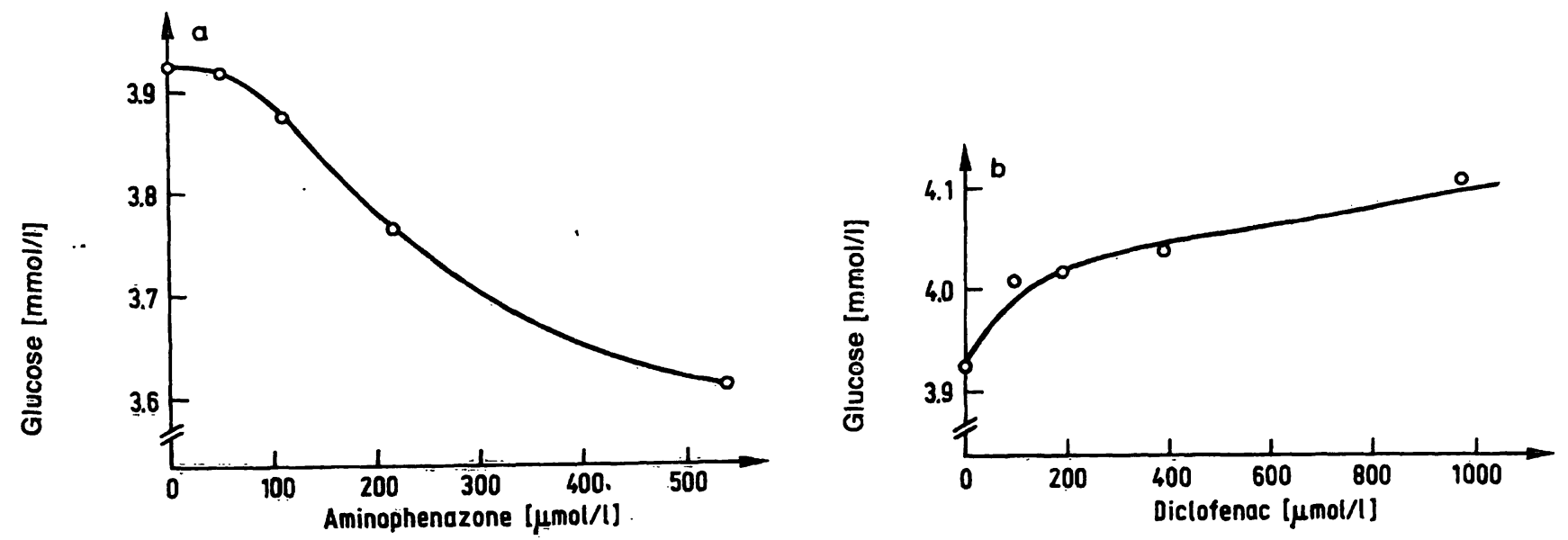

Fig. 1. Dose dependence of aminophenazone (a) and diclofenac (b) interference in the glucose assay with glucose oxidase and peroxidase using ABTS as chromogen. 


\section{References}

1. Jelić, Z., Majkić-Singh, N., Spasić, S., Todorović, P. \& Živanov-Stakić, D. (1984) J. Clin. Chem. Clin. Biochem. 22, 559-563.

2. Jelić-Ivanović, Z., Majkić-Singh, N., Spasić, S., Todorović, P. \& Živanov-Stakić, D. (1983) Clin. Chem. 29, 1859.

3. Siest, G., Appel, W., Blijenberg, G. B., Capolaghi, B., Galteau, M. M., Heusghem, C., Hjelm, M., Lauer, K. L., LePerron, B., Loppinet, V., Love, C., Royer, R. J., Tognoni, C. \& Wilding, P. (1978) J. Clin. Chem. Clin. Biochem. 16, $103-110$.

4. Baselt, R. C., Wright, J.A. \& Cravey, R.H. (1975) Clin. Chem. 21, 44-62.

5. Kaiser, D. G. \& Vangiessen, G. J. (1974) J. Pharm. Sci. 63, 219-221.

6. Riess, W., Stierlin, H., Degen, P., Faigle, J. W., Gerardin, A., Moppert, J., Sallmann, A., Schmid, K., Schweizer, A., Sulz, M., Theobald, W. \& Wagner, J. (1978) Scand. J. Rheumatol., Suppl. 22, 17-29.

7. Upton, R.A., Buskin, J.N., Guentert, T.W., Williams, R. L. \& Riegelman, S. (1980) J. Chromatogr. 190, 119-128.

8. Barthelmai, W. \& Czok, R. (1962) Klin. Wochenschr. 40, $585-590$.

9. Banauch, D., Brümmer, W., Ebeling, W., Metz, H., Rindfrey, H., Lang, H., Leybold, K. \& Rick, W. (1975) J. Clin. Chem. Clin. Biochem. 13, 101-107.

10. Barham, D: \& Trinder, P. (1972) Analyst.97, 142-145.

11. Werner, W., Rey, H. G. \& Wielinger, H. (1970) Z. Analyt. Chem. 252, 224-228.

12. Hyvärinen, A. \& Nikkilä, E. A. (1962) Clin. Chim. Acta 7 , $140-143$.

13. Talke, H. \& Schubert, G. E. (1965) Klin. Wochenschr. 43, 174-179.

14. Fawcett, J. K. \& Scott, J.E. (1960) J. Clin. Pathol. 13, $156-159$.
15. Fearon, W. R. (1939) Biochem. J. 33, 902-907.

16. Morgenstern, S., Flor, R. V., Kaufman, J.H. \& Klein, B. (1966) Clin. Chem. 12, 748-766.

17. Haeckel, R. (1976) J. Clin. Chem. Biochem. 14, 101-107.

18. Kortüm, M. \& Kling, O. (1972) Ärztl. Lab. 18, 33-38.

19. Sobrinho-Simões, M. (1965) J. Lab. Clin. Med. 65, 665-668.

20. Helger, R., Rindfrey, H. \& Hilgenfeldt, J. (1974) J. Clin. Chem. Clin. Biochem. 12, 344-350.

21. Seelig, H.P. \& Wüst, H. (1969) Ärztl. Lab. 15, 34-40.

22. Slot, C. (1965) Scand. J. Clin. Lab. Invest. 4, 381-387.

23. Jendrassik, L. \& Gróf, P. (1938) Biochem. Z. 297, 81-89.

24. Winsten, S. \& Cehelyk, B. (1969) Clin. Chim. Acta 25, $441-446$.

25. Curtius, H.C. \& Roth, M. (eds.) (1974) Clinical Biochemistry, Principles and Methods, W. de Gruyter, Berlin, New York, pp. 1374-1376.

26. Henry, R.J. (1964) Clinical Chemistry, Harper and Row Publ., New York, pp. $181-183$.

27. Richterich, R. (1969) Clinical Chemistry, Theory and Practice, S. Karger, Basel, Academic Press, New York and London, pp. 239-240.

28. Richmond, W. (1965) Clin. Chem. 19, 1350-1356.

29. Watson, D. (1960) Clin. Chim. Acta 5, 637-643.

30. Bucolo, G. \& David, H. (1973) Clin. Chem. 19, 476- 482.

31. Schwarz, D. E. (1957) Bull. Schw. Vereinig. f. Klin. Chem. $1,1-7$.

32. Ramsay, W. N. M. (1953) Biochem. J. 53, 227-231.

33. Young, D. S., Pestaner, L. C. \& Gibberman, V. (1975) Clin. Chem. 21, 1D-432D.

34. Wirth, W.A. \& Thompson, R.L. (1965) Amer. J. Clin. Pathol. 43, 579-591.

35. Caraway, W. T. \& Kammeyer, C.W. (1972) Clin. Chim. Acta 41, 395-434.

Zorana Jelić-Ivanović

Department of Biochemistry

Faculty of Pharmacy

Dr Subotića 8, POB. 146

YU-11000 Beograd 